\title{
Quantitative computed tomography assessment of lung structure and function in pulmonary emphysema
}

\author{
A. Madani, C. Keyzer, P.A. Gevenois
}

Quantitative computed tomography assessment of lung structure and function in pulmonary emphysema. A. Madani, C. Keyzer, P.A. Gevenois. (C)ERS Journals Ltd 2001.

ABSTRACT: Accurate diagnosis and quantification of pulmonary emphysema during life is important to understand the natural history of the disease, to assess the extent of the disease, and to evaluate and follow-up therapeutic interventions.

Since pulmonary emphysema is defined through pathological criteria, new methods of diagnosis and quantification should be validated by comparisons against histological references.

Recent studies have addressed the capability of computed tomography (CT) to quantify pulmonary emphysema accurately. The studies reviewed in this article have been based on CT scans obtained after deep inspiration or expiration, on subjective visual grading and on objective measurements of attenuation values. Especially dedicated software was used for this purpose, which provided numerical data, on both two- and three-dimensional approaches, and compared CT data with pulmonary function tests.

More recently, fractal and textural analyses were applied to computed tomography scans to assess the presence, the extent, and the types of emphysema. Quantitative computed tomography has already been used in patient selection for surgical treatment of pulmonary emphysema and in pharmacotherapeutical trials. However, despite numerous and extensive studies, this technique has not yet been standardized and important questions about how best to use computed tomography for the quantification of pulmonary emphysema are still unsolved.

Eur Respir J 2001; 18: 720-730.

Dept of Radiology, Hôpital Erasme, Université Libre de Bruxelles, Brussels, Belgium.

Correspondence: P.A. Gevenois

Dept of Radiology

Hôpital Erasme

Route de Lennik, 808

1070 Brussels

Belgium

Fax: 3225554388

Keywords: Computed tomography diagnosis

emphysema

Received: December 42000

Accepted after revision June 272001
Computed tomography $(\mathrm{CT})$ is an imaging method providing transverse anatomical images in which the value of each picture element (i.e. pixel) corresponds to the $\mathrm{x}$-ray attenuation of a defined volume of tissue (i.e. voxel). The $\mathrm{X}$-ray attenuation values for each set of projections (i.e. slice) are registered by the computer and organized in a matrix form. The number of calculated pixels determines the image matrix size, influences the image resolution, and should thus be as high as possible. In clinical practice, the matrix size is actually $512 \times 512$ pixels. The $\mathrm{X}$-ray attenuation, also called tissue density, is numerically expressed in Hounsfield units (HU). The scale of attenuation values ranges from $-1,000 \mathrm{HU}$, corresponding to the attenuation value of air, to $3,000 \mathrm{HU}$, with $0 \mathrm{HU}$ corresponding to the attenuation value of water. The thousands of pixels included in one scan make CT the most precise morphological method able to assess the pulmonary structure in vivo [1].

In pulmonary emphysema, the major advantage of $\mathrm{CT}$ is that in addition to providing data concerning overall lung destruction, it also identifies the specific locations in the lung where the alveolar surface has been destroyed. The ability to estimate the extent and severity of pulmonary emphysema during life is important for several reasons. 1) Accurate detection of lung destruction when it appears and careful mapping of its progression are required to understand the natural history of emphysema. 2) The treatment of advanced disease by lung volume reduction surgery (LVRS) requires knowledge of the location of the lesions and objective methods of assessing the surgical

Previous articles in this series: No. 1: Ghaye B, Dondelinger RF. Imaging guided thoracic interventions. Eur Respir J 2001; 17: 507-528. No. 2: Vansteenkiste JF, Stroobants SG. The role of positron emission tomography with ${ }^{18}$ F-fluoro-2-deoxy-D-glucose in respiratory oncology. Eur Respir J 2001; 17: 802-820. No. 3: Kauczor HU, Chen XJ, van Beek EJR, Schreiber WG. Pulmonary ventilation imaged by magnetic resonance: at the doorstep of clinical application. Eur Respir J 2001; 17: 1008-1023. No. 4: Hansell DM. Small airways diseases: detection and insights with computed tomography. Eur Respir J 2001; 17: 1294-1313. No. 5: Franquet T. Imaging of pneumonia: trends and algorithms. Eur Respir J 2001; 18: 196-208. No. 6: Ferretti GR, Bricault I, Coulomb M. Virtual tools for imaging of the thorax. Eur Respir J 2001; 18: 381-392. No. 7: Donkers-van Rossum AB. Diagnostic strategies for suspected pulmonary embolism. Eur Respir J 2001; 18: 589-597. 
results [2]. 3) CT could be a sensitive way of quantitating the progression of emphysema as the determination of the efficacy of replacement therapy in patients with $\alpha_{1}$-antitrypsin deficiency [3]. 4) Studies suggesting that alveolar number and surface-tovolume ratio can be restored by other therapeutic measures in rats with elastase-induced emphysema, imply a future need for measurements that can accurately assess the effectiveness of such therapeutic interventions $[4,5]$. 5) The detection of early emphysema may prevent the occurrence of obstructive ventilatory impairment by smoking cessation or medical intervention [6].

Numerous studies have addressed the capability of CT to accurately quantify the extent and severity of pulmonary emphysema [7, 8]. In order to verify if CT is adequately validated and to suggest possible directions for further studies, this article provides an overview of the previously published studies that were based on quite different methods. Indeed, studies have been based on subjective visual grading or on objective indexes derived from attenuation values, on two-dimensional (2D) or three-dimensional (3D) approaches, and on scans obtained after deep inspiration or expiration.

Since pulmonary emphysema is defined through pathological criteria as an "abnormal permanent enlargement of the airspaces distal to the terminal bronchioles, accompanied by destruction of the alveolar walls, and without obvious fibrosis", new methods of diagnosis and quantification must be validated by comparisons against histopathology [9] but, morphologically, the presence and extent of emphysema can be determined by macroscopic or by microscopic assessment of lung specimen. The most widely used macroscopic and microscopic methods will be briefly reviewed.

\section{Histopathological quantification of pulmonary emphysema}

\section{Macroscopic methods}

Two main methods were traditionally used to macroscopically quantify the severity of emphysema: the point counting developed by DunNILL [10] and the panel grading proposed by THURLBECK et al. [11]. Point counting calculates the proportion, expressed as a percentage, of a lung section occupied by emphysematous spaces by using a transparent plastic sheet with a grid drawn on it and placing it on the lung section. The points of this grid lay $1 \mathrm{~cm}$ apart and are situated at the angles of equilateral triangles with $1 \mathrm{~cm}$ sides. The percentage of the lung involved in emphysema is given by multiplying the number of points superimposed on emphysematous spaces by 100 and dividing by the number of points on the whole lung section. This method is truly quantitative and can be performed on several sections obtained throughout a lung specimen, but it is tedious and time-consuming. The panel grading method is based on the comparison of paper-mounted sagittal lung sections with a set of standards scoring emphysema from $0-100$ at intervals of 5 or 10 . Scores of 5-25 indicate mild emphysema; 30-50, moderate emphysema; and $\geqslant 60$, severe emphysema [11]. This method is quick but not really quantitative, and is a method of ranking emphysema according to several categories of severity. Moreover, this technique underestimates the extent of panlobular emphysema and does not permit combined grading of several sections from the same lung specimen, although it has been shown that an adequate assessment of emphysema cannot be made from a single lung slice [12].

In order to measure the extent of emphysema on numerous paper-mounted lung sections, a computerassisted method following the principles of the point counting method and calculating the relative area, expressed as percentage, of lung macroscopically occupied by emphysema was developed. This method has the advantage of being quick, precise and highly reproducible. It also permits the combination of data from several slices obtained throughout a lung specimen [13].

\section{Microscopic methods}

Several methods have been designed to quantify emphysema microscopically. The mean linear intercept $(\mathrm{Lm})$ is defined as the ratio of the length of a test line placed on a microscopic lung sample divided by the number of intercepts of this test line with alveolar walls [10]. The airspace wall per unit volume (AWUV) is a measurement expressing the alveolar surface area per unit of lung volume and is derived from $\mathrm{Lm}$.

As highlighted by Thurlbeck and Müller [8], neither the loss of alveolar surface nor Lm and AWUV are sensitive methods for recognizing emphysema. Indeed, Lm is normal in $32 \%$ of emphysematous patients [14] and AWUV is abnormal in only $26 \%$ of surgically resected patients with severe macroscopic emphysema [14, 15]. More recently, a computer-based method was used to measure the distance between alveolar walls in the lung parenchyma $[16,17]$. As summarized by THURLBECK and MüLLER [8], "this is a measurement of the average transection distance between walls of alveoli, alveolar ducts, and alveolar sacs considered together; it is not the average alveolar diameter" and this term is less ambiguous than Lm [18].

The destructive index (DI), which is defined as a percentage of destroyed alveolar and alveolar duct space, was introduced as an objective criterion of alveolar wall destruction by SAETTA et al. [19]. DI has three components: breaks in alveolar walls $\left(\mathrm{DI}_{\mathrm{b}}\right)$, metaplasia of type II cells and some degree of fibrosis in alveolar wall $\left(\mathrm{DI}_{\mathrm{f}}\right)$, and the so-called "classic" emphysema $\left(\mathrm{DI}_{\mathrm{e}}\right)$. In the study by SAETTA et al. [19], $\mathrm{DI}_{\mathrm{b}}$ was increased in smokers in whom the size of the airspace was still normal and, therefore, this parameter could be an early indication of lung destruction. Nevertheless, an increased $\mathrm{DI}_{\mathrm{b}}$ could also be related to an increased number and size of fenestrae in the normal parenchyma adjacent to emphysema or to abnormal properties of elastic tissue in smokers without macroscopic emphysema [8]. BoREN [20] reported 
the presence of holes in alveolar walls of normal lung specimen and suggested that holes $>20 \mu \mathrm{m}$ in diameter were abnormal. NAGAI et al. [21] measured the size of these holes and found that only $0.2 \%$ of normal subjects have fenestrae $>20 \mu \mathrm{m}$ in diameter. Alveolar destruction could thus be defined as the presence of holes, also called fenestrae, larger than this diameter, probably the earliest pathological evidence of emphysema [22].

\section{Computed tomography quantification of pulmonary emphysema}

\section{Subjective computed tomography quantification}

Subjective CT quantification of emphysema is based on the visual assessment of areas of vascular disruption and decreased attenuation without clear margins, in comparison with contiguous normal parenchyma [23]. In 1986, BERGIN et al. [24] visually estimated the percentage area that demonstrated changes suggestive of emphysema on contiguous $10-\mathrm{mm}$ thick CT sections, in a study group of 32 patients. On the basis of significant correlations between CT visual scores and macroscopic emphysema graded with a picturegrading system adapted from THURLBECK et al. [11] on midsagittal sections, these authors concluded that CT is a useful adjunct in assessing the presence and extent of emphysema. In 1987, by comparing the CT scores of $1-\mathrm{mm}$ thick high-resolution CT (HRCT) slices performed at five levels of 20 post mortem inflated lung specimens and the pathological scores obtained at the same anatomical levels, HrUBAN et al. [25] demonstrated that HRCT is able to distinguish emphysematous lungs from normal lungs even in the mildest degrees. Applying the same methods in a population of mild emphysematous patients, KuwANo et al. [26] obtained statistically significant correlations between the scores established on the HRCT slices and macroscopic grading, as well as between the CT scores and the microscopic DI. Miller et al. [27] assessed the extent of emphysema by superimposing a grid with squares corresponding to $1 \mathrm{~cm}^{2}$ onto CT images and determining the percentage of squares containing emphysema. Comparing these results, obtained on $10-\mathrm{mm}$ and $1.5-\mathrm{mm}$ thick CT sections, to the grading panel of parasagittal standards established by THURLBECK et al. [11], they found CT insensitive in detecting the earliest lesions of emphysema [27].

In all these studies, i.e. BERgin et al. [24], Hruban et al. [25], KuwANo et al. [26], and Miller et al. [27], "mental adjustments" were required in order to apply the top to bottom grading panel to horizontal images [24-27]. In addition, the grading system is not really quantitative but is a method of ranking emphysema according to categories of severity [11]. To overcome these limitations, a quantitative computer-assisted method was applied to horizontal paper-mounted lung sections producing results on a continuous scale [13]. All these pioneer studies have shown that the extent of emphysema visually scored on CT scans correlates significantly with the extent of emphysema scored on macroscopic lung sections obtained from resected specimen. Most of these studies concerned centrilobular emphysema and were not based on objective CT quantification applied to thin-section CT scans [28].

\section{Objective computed tomography quantification}

Attenuation measurements. In order to objectively quantify pulmonary emphysema, several lung attenuation parameters based on the histogram analysis of the frequency distribution of the attenuation value of the lung, have been developed [29]. The most commonly used methods are based on: the measurement of the mean lung attenuation (MLA); the areas of lung occupied by attenuation values lower than predetermined thresholds [16, 30-34]; and a predetermined percentile of the lung attenuation distribution curve $[3,35]$.

In the first pathological-CT comparative study using numbers for attenuations, HAYHURST et al. [36] showed that the distribution curve of these densities was significantly shifted toward lowest attenuation values in patients with emphysema compared to normal individuals. In a pathological-CT correlation study based on microscopic measurements, Gould et al. [35] showed that the lowest fifth percentile of the histogram of attenuation values was significantly correlated with AWUV. The lowest fifth percentile depends on the extent of emphysema but is also influenced by the relative amount of higher attenuation values, corresponding to airway walls, blood vessels, and any infiltrate that tends to displace the histogram to the right $[37,38]$. Consequently, if emphysema is associated with other pulmonary disorders, the lowest fifth percentile should underestimate the extent of emphysema. To overcome this limitation, an absolute threshold should be used and the relative area of lung occupied by attenuation values lower than this threshold measured [28]. In 1988, MüLLER et al. [32] used a CT commercially available program

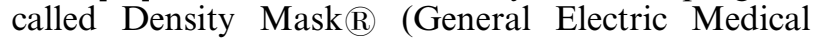
Systems, Milwaukee, WI, USA) that highlights pixels within a given attenuation range and automatically calculates the area of highlighted pixels. In this study, Müller et al. [32] compared the relative area highlighted on a single $1-\mathrm{cm}$ thick CT scan, after injection of contrast material, with the corresponding macroscopic section of the fixed lung cut in the same plane as the CT scan and graded using a modification of the picture-grading system from THURLBECK et al. [11]. The highest correlation was observed with attenuation values $<-910 \mathrm{HU}$, and as consequence, this threshold was recommended for the identification of emphysema. Nevertheless, significant correlations indicate that only CT and pathological scores are statistically linked, but do not imply that the percentage area obtained by CT quantifications are equal to the percentage area occupied by emphysema on the pathological specimen. In addition, the proposed threshold might be influenced by the injection of contrast material. Furthermore, the grading panel does not represent the extent of lung involved in emphysema 
and underestimates panlobular emphysema, especially in the initial stages.

In an attempt to determine the best attenuation threshold for the recognition of emphysema, a program that automatically recognizes the lungs, traces the lung contours, determines histograms of attenuation values, and measures the lung area occupied by pixels included in the predetermined range of attenuation values was applied on 1-mm thick CT sections [39]. On thin CT sections obtained from the lung apices to the base with $1-\mathrm{cm}$ intervals, the relative area of lung, expressed in percentage, occupied by attenuation values lower than various thresholds ranging from -900--970 HU was calculated. In the first study based on 63 patients with lung resection, the CT data was compared with the macroscopic extent of emphysema measured, on horizontal paper-mounted whole lung sections [40], by the authors previously validated computer-assisted method [13]. It was shown that the only threshold for which there was no significant difference between the distribution of the CT measurements and the distribution of macroscopic measurements was $-950 \mathrm{HU}$. Thresholds <-950 HU underestimated emphysema, and thresholds $>-950 \mathrm{HU}$ overestimated emphysema. The case-by-case comparisons between the relative area occupied by attenuation values $<-950 \mathrm{HU}$ (RA950) and the relative area of lung macroscopically occupied by emphysema were not identical in every patient; the mean of the absolute values of the differences between the RA950 and the relative area of lung macroscopically occupied by emphysema was $4.9 \%$ and ranged from $0.1-19.9 \%$. These data suggested that the relative area of lung occupied by attenuation values <-950 HU calculated on thin-section CT scans obtained at full inspiration, was a method allowing an objective quantification of macroscopic emphysema in vivo and with an acceptable error [33].

Since MCLEAN et al. [41] recommended that pulmonary emphysema should be measured microscopically rather than macroscopically, comparisons between CT and morphometry should include microscopic measurements. Using AWUV as a microscopic measurement of the alveolar wall surface in 28 subjects referred for surgical resection of lung tumours, Gould et al. [35] reported significant correlations between AWUV and the lowest fifth percentile of the frequency distribution curve of attenuation values $(\mathrm{r}=-0.77, \mathrm{p}<0.001)$ calculated on $13-\mathrm{mm}$ thick CT sections. In a more recent study based on 38 patients also referred for lung resection, mean interwall distance (MIWD) and mean perimeter (MP) were measured and the percentage surface area of lung occupied by attenuation values lower than thresholds ranging from -900--970 HU was compared to the microscopic indexes. It was shown that the highest correlation was obtained with $-950 \mathrm{HU}(\mathrm{r}=0.70)$. Thus, both the macroscopic and the microscopic study conducted by the authors' group suggested that RA950 is a valuable measurement of the extent of pulmonary emphysema [33].

In order to predict the lung surface to volume ratio from CT attenuation values, Coxson et al. [42] considered $-910 \mathrm{HU}$ as a threshold and compared
CT measurements with histological estimates of surface area. The lung volume was calculated by summing the voxel dimensions in each slice and the lung weight was estimated by multiplying the mean lung attenuation value by the lung volume. From these measurements, Coxson et al. [42] derived the regional lung inflation expressed in $\mathrm{mL} \cdot \mathrm{g}^{-1}$. A comparison of the amount of emphysema detected in the same lobe by both CT and point counting of the resected specimen showed that the volume fraction of lesions $>5 \mathrm{~mm}$ in diameter measured by morphometry is similar to the fraction of lung inflated above $10.2 \mathrm{~mL} \cdot \mathrm{g}^{-1}$. It also showed that lesions $<5 \mathrm{~mm}$ in diameter corresponded to the fraction of the lung inflated between $6.0-10.2 \mathrm{~mL} \cdot \mathrm{g}^{-1}$ and that regions inflated below $6.0 \mathrm{~mL} \cdot \mathrm{g}^{-1}$ are morphologically normal. This method appeared more accurate than the surface area occupied by emphysema since these authors observed a reduced surface to volume ratio in mild emphysema, whereas surface area and tissue weight were decreased only in severe disease.

Comparison between objective and subjective computed tomography quantification

An advantage of computer-assisted quantification is the reproducibility of the technique across readers of varying expertize and experience, and across institutions, allowing more accurate comparison of results among different centres [43]. Conversely, the advantages of a subjective scoring system are the ease of application and no requirement for expensive dedicated software. In a series of 62 patients who underwent thin-section CT prior to surgical lung resection, BANKIER et al. [44] compared subjective visual grading of pulmonary emphysema with macroscopic morphometry and objective CT quantification. Three readers of varying degrees of expertize subjectively graded emphysema in two reading sessions. All three readers systematically overestimated emphysema and the interobserver agreement with weighted Kappa's ranged from $0.431-0.589$. Independent of the level of expertize of the individual reader, the correlation between subjective scores and macroscopic results was weaker than the correlation between objective CT quantification and macroscopic morphological measurements. This study suggests that subjective visual grading should be supplemented with more reliable objective methods whenever a precise and reader-independent quantification of emphysema is required.

\section{Fractal analysis and tissue characterization}

Quantifications of pulmonary emphysema by computer-assisted methods are based on mathematic approaches, namely metrics, that may be used to describe the heterogeneity of the spatial distribution of the attenuation values within the reconstructed image [1]. These metrics include very simple parameters, such as the mean lung density to areas of low attenuation based on single or a range of densities [1]. 
Fractal dimensions are more complex metrics. The concept of fractals, first introduced by MANDELBROT $[45,46]$ is used for a structure with a noninteger number of dimensions. Fractal geometry has found widespread applications in the physical sciences because it is a suitable model for objective quantification of spatial heterogeneity [47]. Unlike Euclidean shape, a fractal cannot be represented by closed-form algebraic relation and continues detail at higher magnifications [47]. A more common definition of a fractal is a shape composed of smaller parts that when enlarged, are similar to the whole shape. Thus, copies of itself can be found at any scale. In summary, the properties of fractals are scale-invariance, selfsimilarity, and fractional dimension.

In order to differentiate normal from emphysematous lungs and normal from emphysematous regions within one lung, UPPALURI et al. [48] developed an adaptive multiple features method (AMFM) based on fractal analysis. With an electron-beam CT scanner, they acquired 2D slices of the whole lung with 3-mm collimation at maximal inspiration. Adjacent pixels with small differences in gray level were merged and first- as well as second-order statistical measurements were separately computed for each slice. First-order measurements include mean, variance and skewness of the attenuation distribution curve, whereas secondorder measurements include co-occurrence entropy, contrast, angular second moment, etc. These authors observed that second-order statistics and fractal dimension were sensitive to the gray level and spatial relationships between pixels in a region. These parameters can thus be used for tissue characterization. These authors compared AMFM, mean lung density, and the lowest fifth percentile of the distribution histogram of attenuation values to discriminate normal from emphysematous lungs. The accuracy of AMFM, mean lung density, and the lowest fifth percentile was $100 \%, 95 \%$, and $97 \%$, respectively. However, there was no correlation between these three parameters and pulmonary function tests (PFTs). These authors explained this lack of correlation by a too few number of slices per subject and by the absence of patients with mild and moderate emphysema included in their study [1, 49].

More recently, Mishima et al. [30] attempted to detect early emphysema on the basis of fractal analysis. They quantified the size distribution of low attenuation area (i.e. <-960 HU) clusters on 2-mm thick HRCT slices obtained at full inspiration in healthy subjects $(n=30)$ and chronic obstructive pulmonary disease (COPD) patients $(n=73)$. All normal subjects had a low attenuation area $<30 \%$ of the total lung area, which varied from $2.6-67.6 \%$ in COPD patients. These authors observed that the cumulative size distribution of the low attenuation area clusters followed a power-law distribution characterized by an exponent D. Although the COPD group of patients with a low attenuation area $<30 \%$ of total lung area and the normal subjects had similar low attenuation areas, the corresponding $\mathrm{D}$ values were significantly smaller in the COPD patients. On the basis of an elastic spring network model, these authors attributed this smaller value to the coalescence of smaller low attenuation area clusters into larger low attenuation area clusters in COPD patients. There was no correlation between the value of D and PFTs, with the exception of the carbon monoxide diffusing capacity of the lung $(D \mathrm{~L}, \mathrm{CO})$. Assuming that the exponent $\mathrm{D}$ is related to the fractal dimension of the alveolar surface (df), a measure of terminal airspace geometry complexity, a smaller D in a 2D CT image is the consequence of larger low attenuation area clusters and represents the reduced $\mathrm{df}$ in COPD patients. These authors concluded that $30 \%$ could be the critical value of low attenuation area to discriminate normal and mild from severe COPD patients, but that low attenuation area is not sufficiently accurate to distinguish early emphysematous patients from normal subjects. The value of $\mathrm{D}$ could be a sensitive parameter in order to detect terminal airspace enlargement that occurs in early emphysema.

\section{Factors influencing computed tomography densito- metry}

Age. Morphometric data from Thurlbeck [50] and Gillooly and LAMB [51] showed a significant correlation between airspace size and age. The increase of airspace size associated with advanced age could thus influence the CT density parameters and should be taken into account for longitudinal studies. In order to investigate the possible influence of age on density measurements, the RA950 was measured in 42 healthy subjects aged 23-71 yrs and a weak but significant correlation between age and the RA950 ( $\mathrm{r}=0.328$; $\mathrm{p}=0.034$ ) was found. These results are in accordance with those from SoEIJIMA et al. [52] who investigated 36 symptom-free nonsmoking subjects with normal lung function during a 5-yr follow-up period and showed that the percentage of RA960 increased with age, at least in the middle and lower lung zones.

Computed tomography parameters. Since CT scanning parameters could influence the attenuation values and their distribution curve, Mishima et al. [53] compared the low attenuation area $(<-960 \mathrm{HU})$ obtained with a various number of slices ranging from 3-10, various slice thicknesses from $2-5 \mathrm{~mm}$, and various electric tube currents ranging from $50-250 \mathrm{~mA}$. On the basis of image quality, exposure dose, and correlations with lung function tests, they suggested that three 2-mm thick CT sections, acquired with $200 \mathrm{~mA}$ tube current, are the most appropriate parameters to assess pulmonary emphysema.

Number of computed tomography sections. Pulmonary emphysema is heterogeneously distributed throughout the lung, and from studies based on point-counting, it is known that an adequate assessment cannot be obtained from one lung slice alone [12], but radiation exposure is likely to favour sampling techniques rather than whole lung measurements. Depending on the presence of emphysema and on its spatial distribution, the minimum number of scans providing accurate results could change from patient-to-patient, but no CT study has defined the minimum number of scans 
necessary to provide accurate results. In the study based on comparisons between HRCT and macroscopy, attempts were made to define a maximum interval distance between HRCT scans providing valid results. The RA950 was recalculated by successively considering one scan of two, one of three, one of four and so on, and comparing these results to the results obtained with $1 \mathrm{~cm}$ intervals. The individual variability of the RA950 was very heterogeneous from patient-topatient and no bend in the relationship linking the coefficient of variation of RA950 and the interval distance was found. Consequently, no particular interval distance could be proposed as an optimal standard [33]. More recently, Mishima et al. [53] attempted to define the influence of the slice number on RA960 in 30 patients with COPD. These authors calculated correlation coefficients between RA960 measured on 5, 3, and 2 CT sections and RA960 measured on 10 CT sections. The corresponding coefficients were 0.976, 0.953 , and 0.908 , indicating highly significant correlations. The authors concluded that three slices were sufficient to obtain the overall extent of emphysema but they did not report the severity of the disease in terms of functional deficit or the heterogeneity of its spatial distribution within the lungs.

Spiral computed tomography. Spiral CT scanning has the major advantage that the entire thorax is imaged during one single breath-hold. This method involves simultaneous transport of the patient at a constant speed through the CT gantry while data are continuously acquired over multiple gantry rotations [54]. From spiral CT data, 3D reconstructions, lung volume measurements, and quantification of lung disorders can be obtained. By using dedicated software that are currently available on almost all modern scanners, KAUCZOR et al. [55] compared lung volumes measured by spiral CT and plethysmography. They observed significant correlations between both measurements and an underestimation of the total lung capacity (TLC) by $12 \%$, measured by spiral CT, probably due to the supine posture of the subject in the CT scanner compared to the seated posture in the plethysmograph. Spiral CT data could be of great interest in the quantification of heterogeneously distributed lung disorders such as pulmonary emphysema, but no study has validated spiral CT parameters as compared to histopathology. Dedicated programs reconstructing a 3D model of the lungs, calculating their volume and providing the frequency distribution curve of attenuation values within this lung volume can be applied to spiral CT data. Such a program has been used by PARK et al. [56] who compared the percentage volume of the lung occupied by attenuation values lower than three thresholds (-900 HU, -910 HU, -950 HU) with the percentage area of the lung occupied by attenuation values lower than these thresholds. They observed highly significant correlations $(r=\sim 0.98)$ between lung attenuation measurements obtained with the 3D model and those from 2D images.

Lung volume and size. The possible role of CT obtained after deep expiration in the assessment of emphysema was first suggested by KNUDSON et al. [57] because correlations between CT measurements and lung function tests were stronger in expiration than in inspiration. In 64 patients, most of whom had airflow obstruction, these authors obtained 8-mm thick CT slices at two levels in upper lung zones at full inspiration and full expiration and measured the percentage area of lung occupied by attenuation values <-900 HU. This percentage area was compared with various PFTs, such as static lung compliance, DL,CO, and forced expiratory volume in one second (FEV1). The highest correlations between CT with physiological variables consistent with emphysema, were observed with CT measurements obtained at full expiration.

In order to investigate the possible role of quantitative CT during expiration, the relative areas of lung occupied by attenuation values lower than various thresholds ranging from -800--970 HU, at full inspiration and full expiration, in 89 patients who underwent surgical resection were measured. Two different thresholds were found, valid by comparisons with macroscopy (-910 HU) and microscopy (-820 HU), and they were quite different from the threshold values found to be valid for CT scans obtained at full inspiration (-950 HU) [16, 33]. In addition, multiple regression analysis showed that CT measurements obtained at full expiration did not yield any additional significant information compared to those obtained at full inspiration in order to predict the anatomical extent of emphysema. In a study based on visual scoring, NishimuRA et al. [58] showed that expiratory CT underestimates the degree of emphysema as compared with inspiratory CT scans. In summary, expiratory $\mathrm{CT}$ is thus not as adequate as inspiratory CT for measuring the extent of pulmonary emphysema. This conclusion suggests that possible errors, secondary to variations of lung volume at which the CT scans are obtained, could be avoided by using spirometric triggering [53-55, 59].

In the authors' study on expiratory CT, correlations between PFTs and objective CT data respectively obtained in deep expiration and in deep inspiration, respectively, were investigated [17]. Higher correlations between PFTs reflecting the airflow obstruction and the CT data obtained in expiration than in inspiration were observed but correlations were similar between diffusing capacity and both inspiratory and expiratory data. It was concluded that expiratory CT reflects expiratory airflow limitation and the subsequent air trapping more than reduction of the alveolar wall surface. These findings were confirmed by EDA et al. [59] and by LAMERs et al. [60] who also found higher correlations between PFTs reflecting the airflow obstruction and CT data obtained in expiration than in inspiration, whereas the correlations between diffusing capacity and CT data were similar.

Independent of the lung volume at which the CT scan is obtained, the lung size could influence CT parameters. Morphometric studies showed contradictory results, suggesting either that the number of alveoli in the human lung was [61-63] or was not positively correlated with body length [64]. In the authors' cross-sectional study on 42 healthy subjects, a significant correlation was found between TLC and 
the MLA ( $\mathrm{r}=-0.419 ; \mathrm{p}=0.006)$ as well as between TLC and the RA950 $(\mathrm{r}=0.386 ; \mathrm{p}=0.012)$. The larger the TLC in absolute value, the lower the MLA and the higher the RA950. These results suggest that the relative amount of lung tissue per unit of volume is lower in larger lungs than in smaller lungs. According to this, because the structure of the alveolar wall is unrelated to the lung size, the dimensions of the airspaces should be greater in larger lungs than in smaller lungs.

\section{Comparison between computed tomography quantifica- tion and pulmonary function tests}

Although PFTs may be short- and long-term reproducible tests, they represent global measurements of lung function of more than 10 million airways that contribute unequally to airflow [65]. They are of limited value in the measurement of the obstruction of airways, particularly small airways that are predominantly affected in emphysema [65] and autopsy studies have shown that up to one-third of the lung can be destroyed by emphysema before respiratory function becomes impaired [48]. The insensitivity of PFTs to diagnose mild emphysema was confirmed by SANDERS et al. [66] who found that emphysematous features of emphysema were visually detected on CT scans in $69 \%$ of smokers with normal $D$ L,CO with or without associated obstructive deficit. In this study, CT showed evidence of emphysema in $96 \%$ of patients selected on the basis of functional criteria of emphysema as suggested by the American Thoracic Society, such as decreased DL,CO $(<80 \%$ predicted value), and plus evidence of obstructive lung disease (decreased FEV1 $<80 \%$ and/or increased residual volume (RV) $>120 \%$ pred). These authors concluded that CT may be more sensitive than PFTs in detecting mild emphysema.

The lack of sensitivity of PFTs to detect pulmonary emphysema can be explained by two reasons, which are related to pulmonary zones in which ventilatory disorders are not assessed by conventional PFTs. First, the total airflow resistance of all respiratory bronchioles contributes little to the total airflow resistance of the lung [65]. Despite the high airflow resistance through one single respiratory bronchiole, the parallel connection of a high number of bronchioles leads to a wide total cross-sectional area and drastically reduces the airflow resistance [37]. Second, the upper lung zone has a relatively high ventilation/ perfusion $\left(V^{\prime} / Q^{\prime}\right)$ ratio compared to lower lung zone. Thus, in the relatively underventilated upper lung zone, emphysema produces smaller measurable pulmonary dysfunction than in the lower zone. Consistently, Gurney et al. [67] and Haragushi et al. [68] showed that the extent of emphysema had higher correlations with $D \mathrm{~L}, \mathrm{CO}$ in the lower lung zone than in the upper lung zone, even though the upper lung zone was more severely affected by emphysema. On the basis of the lobar distribution of emphysema as determined by CT, SAITOH et al. [69] reported that the airflow limitation, the RV and the TLC were higher in the predominantly lower-lobe emphysematous group than in the predominantly upper-lobe emphysematous group. However, NAKANO et al. [34] reported that the correlations between the RA960, and FEV1 or RV/TLC were higher in the lower lobes but the correlation between RA960 and DL,CO was higher in the upper lobes. The authors attributed this discrepancy between their results and the results of the other studies, to the high incidence of severe emphysema in the upper lobe, which affects the $D$ L,CO. The central versus peripheral predominant location of emphysematous area also determines the importance of functional impairment. HARAGUSHI et al. [68] found higher correlations between PFTs, including FEV1 and $D \mathrm{~L}, \mathrm{CO}$, and attenuation values of the central region than the peripheral region of lungs. This is in agreement with the study of NAKANO et al. [34], which reported a higher incidence of emphysema in central region compared to peripheral region. These authors explained the results by the move of particle deposition from outer to inner lung, by a greater stratified distribution of pulmonary blood perfusion in the outer than in the inner lung, and by the lymphatic drainage of particles from outer to inner lung, which is favoured by the ventilatory movements [34].

The correlation between CT indexes and DL,CO or $D$ L,CO/alveolar volume ( $V \mathrm{~A})$ were extensively documented in numerous studies and ranged from -0.5-0.75 [35, 70-73]. In the authors' study, comparing CT with microscopic morphometry, correlation coefficients between RA950 and microscopic indexes of the same degree of magnitude as between FEV1/VC or $D \mathrm{~L}, \mathrm{CO} / V \mathrm{~A}$ and these microscopic indexes $(\mathrm{r}=\sim 0.70)$ were obtained. Considering the microscopic measurements of the method of reference, the RA950 and independent PFTs in stepwise procedures were entered. The relationships obtained revealed that $D \mathrm{~L}, \mathrm{CO}$ and the RA950 were sufficient to predict microscopic measurements [16].

More recently, using the 3D model described earlier, PARK et al. [56] investigated the relationships between PFTs and the percentage of lung volume occupied by attenuation values lower than three thresholds (-900 HU, -910 HU, -950 HU), and found moderate-to-high correlations between these percentage volumes and TLC ( $\mathrm{r}=0.62-0.71)$, FEV1 ( $\mathrm{r}=$ $-0.57-0.60)$ and $\mathrm{FEV} 1 / \mathrm{FVC}(\mathrm{r}=-0.75-0.82)$, and $D$ L,CO (r=-0.57-0.64) [64]. The percentage volume $<-950$ HU correlated more closely with $D$ L,CO and FEV1 than the volumes <-910HU and $-900 \mathrm{HU}$. The authors concluded that lung densitometry derived from 3D lung models is an alternative method for quantifying emphysema.

\section{Surgical treatment of emphysema}

LVRS is a therapeutic option for severe debilitating emphysema consisting of bilateral wedge resection of emphysematous lung by means of sternotomy, bilateral thoracotomy, or video-assisted thoracoscopic surgery [43]. This technique induces a one-time benefit improvement, peaking at 3-6 months after surgery, in terms of lung function, exercise tolerance, and quality of life [74-77]. The retrospective nature of patient selection and the inability to accurately quantify the 
amount of resected emphysematous lung tissue are two major obstacles in defining criteria for candidate's selection for LVRS [75]. Patients are usually selected on the basis of clinical, physiological and radioanatomical assessments. Intolerable dyspnoea and exercise intolerance not palliated by medical therapy, and severe airflow obstruction are the main clinical criteria. Conversely, anatomical features, consisting of lobar severity of emphysema, with a heterogeneous distribution of emphysema, are important for a better clinical outcome. Upper lobe predominance, a higher amount of regional heterogeneity, and a larger percentage of normal or mildly emphysematous lung showed the highest association with improvement of quality of life and exercise tolerance [43].

In a study based on visual CT analysis of emphysema, WEDER et al. [78] showed that the mean increase in FEV1 after LVRS was $\sim 80 \%, 40 \%$, and $35 \%$ for heterogeneous, intermediately heterogeneous and homogeneous emphysema, respectively. However, this study revealed that preoperative characteristics of pulmonary function or chest CT morphology could not explain the postoperative improvement in terms of FEV1.

In order to determine whether quantitative CT provides relevant information for guiding patient selection, GierADA et al. [2] compared CT quantification of pulmonary emphysema with FEV1, arterial oxygen tension $\left(\mathrm{Pa}_{\mathrm{a}} \mathrm{O}_{2}\right)$, and 6-min walking distance before and after LVRS. They showed that the values of quantitative CT indexes of global and regional emphysema severity were related to outcome measures after LVRS. Indexes of global emphysema severity include the MLA, the percentage of whole lung with attenuation <-900 HU (emphysema index) and the percentage of whole lung with attenuation $<-960 \mathrm{HU}$ (severe emphysema index). Indexes of regional emphysema severity include emphysema indexes in the upper and lower halves of the lungs and the ratio of the emphysema index of the upper lung to that of lower lung. Postoperative improvement was better with an MLA >-900 HU, an emphysema index of $\geqslant 75 \%$, a severe emphysema index $>25 \%$, a ratio of upper- and lower-lung emphysema indexes $\geqslant 1.5$ and an upper lung emphysema index $>75 \%$. When considering other simple characteristics of the attenuation distribution curve, such as the standard deviation and the full width at half maximum, the authors did not identify a CT index of emphysema heterogeneity predictive of patient outcome.

As recently reviewed by KAzEROONI [43], a high upper- and lower-lung emphysema ratio has been the best predictor of improvement in terms of FEV1 and 6-minute walk distance for 2 yrs after bilateral apical LVRS. This ratio demonstrated a higher correlation with outcome than the percentage of emphysema of the whole lung and the functional parameters reflecting hyperinflation, such as RV, TLC, RV/TLC ratio and FEV1, or DL,CO [43]. Quantitative CT could play an important role in identifying potentially suitable candidates and standardizing the preoperative imaging evaluation of these candidates.
New perspectives in pharmacotherapy

For many years it was hypothesized that in patients with $\alpha_{1}$-antitryspsin deficiency, replacement therapy could prevent the progression of pulmonary emphysema. In a double-blind controlled study performed over a 3-yr period and involving 56 patients with $\alpha_{1}$-antitryspsin deficiency of PI $* Z Z$ phenotype and moderate-to-severe emphysema, DIRKSEN et al. [3] estimated the loss of lung tissue by calculating the 15 th lowest percentile of the attenuation distribution curve. They found that this loss tended to be higher in the group of untreated patients as compared with the group of patients treated by $\alpha_{1}$-antitryspsin infusion $(p=0.07)$. In addition, power analysis showed that this protective effect would reach statistical significance in a similar trial with 130 patients, in contrast to calculations based on annual decline of FEV1 showing that 550 patients would be needed to show a $50 \%$ reduction on annual decline. These authors concluded that lung density measurements could be more sensitive than PFTs in detecting the progression of emphysema and that CT may facilitate future randomized clinical trials.

\section{Conclusion}

CT scanning is of particular interest for the in vivo diagnosis and quantification of pulmonary emphysema because this imaging technique offers measurements of both morphological and functional information with the possibility of interrelating structure and function. The presence and extent of pulmonary emphysema can be roughly estimated by visual assessment of CT sections, but objective quantification is more accurate and more reproducible. This review shows the possible important role of CT in the diagnosis and quantification of pulmonary emphysema. Nevertheless, this technique is not yet standardized and important questions are still unsolved. Further studies are needed for the following purposes: 1) to establish normal values of reference; 2) to investigate the reproducibility of CT measurements; 3 ) to investigate the influence of image acquisition parameters on the CT measurements; 4) to compare the accuracy of various CT indexes based on a percentile of the frequency distribution curve, on absolute thresholding, on regional lung inflation, or on a fractal dimension; 6) to evaluate the accuracy of CT in quantifying as well as diagnosing pulmonary emphysema and in recognizing subtypes of emphysema; 7) to validate new CT techniques, such as multidetector systems; and 8) to investigate the ability of CT to discriminate emphysema from other COPD.

Since it is now well accepted that computed tomography during inspiration allows reasonably accurate objective quantification of the extent of pulmonary emphysema, the computed tomography techniques should be standardized. 


\section{References}

1. Hoffman EA, McLennan G. Assessment of the pulmonary structure-function relationship and clinical outcomes measures. Acad Radiol 1997; 4: 758-776.

2. Gierada DS, Slone RM, Bae KT, Yusen RD, Lefrak SS, Cooper JD. Pulmonary emphysema: comparison of preoperative quantitative CT and physiologic index values with clinical outcome after lung volume reduction surgery. Radiology 1997; 205: 235-242.

3. Dirksen A, Dijkman JH, Madsen F, et al. A randomized clinical trial of $\alpha_{1}$-antitrypsin augmented therapy. Am J Respir Crit Care Med 1999; 160: 1468 1472.

4. Tepper J, Pfeiffer J, Aldrich M, et al. Can retinoic acid ameliorate the physiologic and morphologic effects of elastase instillation in the rat? Chest 2000; 117: 242S244S.

5. Stockley RA. Alpha-1-antitrypsin deficiency: what next? Thorax 2000; 55: 614-618.

6. Morgan MDL. Detection and quantification of pulmonary emphysema by computed tomography: a window of opportunity. Thorax 1992; 47: 1001-1004.

7. Stern EJ, Franck MS. CT of the lung in patients with pulmonary emphysema: diagnosis, quantification, and correlation with pathologic and physiologic findings. AJR 1994; 162: 791-798.

8. Thurlbeck WM, Müller NL. Emphysema: definition, imaging, and quantification. AJR 1994; 163: 10171025 .

9. Snider GL, Kleinerman JL, Thurlbeck WM, Bengali ZH. The definition of emphysema. Report of a national Heart, Lung, and Blood Institute, Division of Lung Disease Workshop. Am Rev Respir Dis 1985; 132: 182-185.

10. Dunnill MS. Quantitative methods in the study of pulmonary pathology. Thorax 1962; 17: 320-328.

11. Thurlbeck WM, Dunnill MS, Hartung W, Heard BE, Heppleston AG, Ryder RC. A comparison of three methods measuring emphysema. Hum Pathol 1970; 1: 215-226.

12. Turner P, Whimster WF. Volume of emphysema. Thorax 1981; 36: 932-937.

13. Gevenois PA, Zanen J, de Maertelaer V, De Vuyst P, Dumortier P, Yernault JC. Macroscopic assessment of pulmonary emphysema by image analysis. $J$ Clin Pathol 1995; 48: 318-322.

14. Thurlbeck WM. Internal surface area and other measurements in emphysema. Thorax 1967; 22: 483 496.

15. Gillooly M, Lamb D. microscopic emphysema in relation to age and smoking habit. Thorax 1993; 48: 491-495.

16. Gevenois PA, De Vuyst $\mathrm{P}$, de Maertelaer V, et al. Comparison of computed density and microscopic morphometry in pulmonary emphysema. Am J Respir Crit Care Med 1996; 154: 187-192.

17. Gevenois PA, De Vuyst P, Sy M, et al. Pulmonary emphysema: quantitative CT during expiration. Radiology 1996; 199: 825-829.

18. Müller NL, Thurlbeck WM. Thin-section CT, emphysema, air-trapping, and airway obstruction. Radiology 1996; 199: 621-622.

19. Saetta M, Shiner RJ, Angus GE, et al. Destructive index: a measurement of lung parenchyma destruction in smokers. Am Rev Respir Dis 1985; 131: 764-769.

20. Boren HG. Alveolar fenestrae: relationship to the pathology and pathogenesis of pulmonary emphysema. Am Rev Respir Dis 1962; 85: 328-344.

21. Nagai A, Inano $H$, Matsuba $K$, Thurlbeck WM. Scanning electronmicroscopy morphometry of emphysema in humans. Am J Respir Crit Care Med 1994; 150: $1411-1415$.

22. Cosio MG, Shiner RJ, Saetta M, et al. Alveolar fenestrae in smokers. Relationships with light microscopic and functional abnormalities. Am Rev Respir Dis 1986; 133: 126-131.

23. Bergin CJ, Muller NL, Miller RR. CT in the quantitative assessment of emphysema. $J$ Thorac Imaging 1986; 1: 94-103.

24. Bergin C, Müller N, Nichols DM, et al. The diagnosis of emphysema: a computed tomographic-pathologic correlation. Am Rev Respir Dis 1986; 133: 541-546.

25. Hruban RH, Meziane MA, Zerhouni EA, et al. High resolution computed tomography of inflation-fixed lungs: pathologic-radiologic correlation of centrolobular emphysema. Am Rev Respir Dis 1987; 136: 935-940.

26. Kuwano K, Matsuba K, Ikeda T, et al. The diagnosis of mild emphysema: correlation of computed tomography and pathology scores. Am Rev Respir Dis 1990; 141: 169-178.

27. Miller RR, Muller NL, Vedal S, Morrison NJ, Stapels CA. Limitations of computed tomography in the assessment of emphysema. Am Rev Respir Dis 1989; 139: 980-983.

28. Gevenois PA, Yernault JC. Can computed tomography quantify pulmonary emphysema? Eur Respir $J$ 1995; 5: 843-848.

29. Stoel BC, Vrooman HA, Stolk J, Reiber JHC. Sources of error in lung densitometry with CT. Invest Radiol 1999; 34: 303-309.

30. Mishima M, Hirai $\mathrm{T}$, Itoh $\mathrm{H}$, et al. Complexity of terminal airspace geometry assessed by lung computed tomography in normal subjects and patients with chronic obstructive pulmonary disease. Proc Natl Acad Sci 1999; 96: 8829-8834.

31. Bae KT, Slone RM, Gierada DS, Yusen RD, Cooper JD. Patients with emphysema: quantitative CT analysis before and after lung volume reduction surgery. Radiology 1997; 203: 705-714.

32. Müller NL, Stapels CA, Miller RR, Abboud RJ. "Density Mask": an objective method to quantitate emphysema using computed tomography. Chest 1988; 94: 782-787.

33. Gevenois PA, de Maertelaer V, De Vuyst P, Zanen J, Yernault JC. Comparison of computed density and macroscopic morphometry in pulmonary emphysema. Am J Respir Crit Care Med 1995; 152: 653-657.

34. Nakano Y, Sakai H, Hirai T, Oku Y, Nishimura K, Mishima M. Comparison of low attenuation areas on computed tomographic scans between inner and outer sefments of the lung in patients with chronic obstructive pulmonary disease: incidence and contribution to lung function. Thorax 1999; 54: 384-389.

35. Gould GA, Macnee W, McLean A, et al. CT measurements of lung density in life can quantitate distal airspace enlargement: an essential defining feature of human emphysema. Am Rev Respir Dis 1988; 137: 380-392.

36. Hayhurst MD, Flenley DC, McLean A, et al. Diagnosis of pulmonary emphysema by computerised tomography. Lancet 1984; 2: 320-322.

37. Rienmüller RK, Behr J, Kalender A, et al. Standardized 
quantitative high resolution $\mathrm{CT}$ in lung diseases. J Comput Assist Tomogr 1991; 15: 742-749.

38. Hartley PG, Galvin JR, Hunninghake GW, et al. High-resolution CT-derived measures of lung density are valid indexes of interstitial lung diseases. $J$ Appl Physiol 1994; 76: 271-277.

39. Kalender WA, Fitche H, Bautz W, Skalej M. Semiautomatic evaluation procedure for quantitative CT of the lung. J Comput Asisst Tomogr 1991; 15: 248-255.

40. Gevenois PA, Koob MC, Jacobovitz D, De Vuyst P, Yernault JC, Struyven J. Whole lung sections for computed tomographic-pathologic correlations. Modified Gough-Wentworth technique. Invest Radiol 1993; 28: 242-246.

41. McLean A, Warren PM, Gillooly M, MacNee W. Microscopic and macroscopic measurements of emphysema: relation to carbon monoxide gas transfer. Thorax 1992; 47: 144-149.

42. Coxson HO, Rogers RM, Whittall KP, et al. A quantification of the lung surface area in emphysema using computed tomography. Am J Respir Crit Care Med 1999; 159: 851-856.

43. Kazerooni EA. Radiologic evaluation of emphysema for lung volume reduction surgery. Clin Chest Med 1999; 20: 845-861.

44. Bankier AA, de Maertelaer V, Keyzer C, Gevenois PA. CT of pulmonary emphysema: subjective assessment and objective quantification by densitometry and macroscopic morphometry. Radiology 1999; 211: 851-858.

45. Mandelbrot BB. Fractals: Form, Chance, and Dimension. San Francisco, Freeman, 1997.

46. Mandelbrot BB. The Fractal Geometry of Nature. San Francisco, Freeman, 1982.

47. McNamee JE. Fractal perspectives in pulmonary physiology. J Appl Physiol 1991; 71: 1-8.

48. Uppaluri R, Mitsa T, Sonka M, Hoffman EA, McLennan G. Quantification of pulmonary emphysema from lung computed tomography images. Am J Respir Crit Care Med 1997; 156: 248-254.

49. Rodarte JR, Chaniotakis M, Wilson TA. Variability of parenchymal expansion measured by computed tomography. J Appl Physiol 1989; 67: 226-231.

50. Thurlbeck WM. The internal surface area of nonemphysematous lungs. Am Rev Respir Dis 1967; 95: 765-773.

51. Gillooly M, Lamb D. Airspace size in lungs of lifelong non-smokers: effect of age and sex. Thorax 1993; 48: $39-43$.

52. Soeijima K, Yamaguchi K, Kohda E, et al. Longitudinal follow-up study of smoking-induced lung density changes by high resolution computed tomography. Am J Respir Crit Care Med 2000; 161: 1264 1273.

53. Mishima $\mathrm{M}$, Itoh $\mathrm{H}$, Sakai $\mathrm{H}$, et al. Optimized scanning conditions of high resolution $\mathrm{CT}$ in the follow-up of pulmonary emphysema. J Comput Assist Tomogr 1999; 23: 380-384.

54. Kohz P, Stäbler A, Beinert T, et al. Reproductibility of quantitative, spirometrically controled CT. Radiology 1995; 197: 539-542.

55. Kauczor HU, Heussel CP, Fisher B, Klamm R, Mildenberger $P$, Thelen $M$. Assessment of lung volumes using helical $\mathrm{CT}$ at inspiration and expiration: comparison with pulmonary function tests. $A J R$ 1998; 171: 1091-1095.

56. Park KJ, Bergin CJ, Clausen JL. Quantification of emphysema with three-dimensional CT densitometry: comparison with two-dimensional analysis, visual emphysema scores, and pulmonary function test results. Radiology 1999; 211: 541-547.

57. Knudson RJ, Standen JR, Kaltenborn WT, et al. Expiratory computed tomography for assessment of suspected pulmonary emphysema. Chest 1991; 99: 1357-1366

58. Nishimura K, Murata K, Yamagishi M, et al. Comparison of different computed tomography scanning methods for quantifying emphysema. $J$ Thorac Imag 1998; 13: 193-198.

59. Eda S, Kubo K, Fujimoto K, Matsuzawa Y, Sekiuchi M, Sakai F. The relations between expiratory chest CT using helical scanning and pulmonary function tests in emphysema. Am J Respir Crit Care Med 1997; 155: 1290-1294.

60. Lamers RJ, Thelissen GR, Kessels AG, Wouters EF, Engelshoven JM. Chronic obstructive pulmonary disease: evaluation with spirometrically controlled CT lung densitometry. Radiology 1994; 193: 109-113.

61. Dunnill MS. Postnatal growth of the lung. Thorax 1962; 17: 329-333.

62. Dunnill MS. Evaluation of a simple method of sampling the lung for quantitative histological analysis. Thorax 1964; 19: 443-448.

63. Weibel ER. Morphometry of the Human Lung. Berlin, Springer, 1963.

64. Angus GE, Thurlbeck WM. Number of alveoli in the human lung. J Appl Physiol 1972; 32: 483-485.

65. Gurney JW. Pathophysiology of obstructive airways disease. Radiol Clinic North Am 1998; 36: 15-27.

66. Sanders C, Nath PH, Bailey WC. Detection of emphysema with computed tomography: correlation with pulmonary function tests and chest radiography. Invest Radiol 1988; 23: 262-266.

67. Gurney JW, Jones KK, Robbins RA, et al. Regional distribution of emphysema: correlation of high-resolution CT with pulmonary function tests in unselected smokers. Radiology 1992; 183: 457-463.

68. Haragushi M, Shimura S, Hida W, Shirato K. Pulmonary function and regional distribution of emphysema as determined by high-resolution computed tomography. Respiration 1998; 65: 125-129.

69. Saitoh T, Koba H, Shijubo N, Tanaka H, Sugaya F. Lobar distribution of emphysema in computed tomographic densitometric analysis. Invest Radiol 2000; 35 : 235-243.

70. Morrison NJ, Abboud RT, Ramadan F, et al. Comparison of single breath carbon monoxide diffusing capacity and pressure-volume curves in detecting emphysema. Am Rev Respir Dis 1989; 139: 1179-1187.

71. Kinsella M, Müller NL, Abboud RT, Morrison NJ, DyBuncio A. Quantitation of emphysema by computed tomography using a "Density Mask" program and correlation with pulmonary function tests. Chest 1990; 97: 315-321.

72. Watanuki Y, Shunsuke S, Nishikawa M, Miyashita A, Okubo T. Correlation of quantitative CT with selective alveolobronchogram and pulmonary function tests in emphysema. Chest 1994; 106: 806-813.

73. Gelb AF, Hogg JC, Müller NL, et al. Contribution of emhysema and small airways in COPD. Chest 1996; 109: 353-359.

74. Cooper JD, Lefrak SS. Is volume reduction surgery appropriate in the treatment of emphysema? Yes. Am J Respir Crit Care Med 1996; 153: 1201-1204. 
75. Russi EW, Bloch KE, Weder W. Functional and morphological heterogeneity of emphysema and its implication for selection of patients for lung volume reduction surgery. Eur Respir J 1999; 14: 230-236.

76. Gelb AF, Brenner M, McKenna RJ Jr, Fischel R, Zamel N, Schein MJ. Serial lung function and elastic recoil 2 years after lung volume reduction surgery for emphysema. Chest 1998; 113: 1497-1506.
77. Geddes D, Davies M, Koyama H, et al. Effect of lungvolume reduction surgery in patients with severe emphysema. N Engl J Med 2000; 343: 239-245.

78. Weder W, Thurnheer R, Stammberger U, Bürge M, Russi EW, Bloch KE. Radiological emphysema morphology is associated with outcome after surgical lung volume reduction. Ann Thorac Surg 1997; 64: 313-320. 